\title{
Video Article \\ Integrating Augmented Reality Tools in Breast Cancer Related Lymphedema Prognostication and Diagnosis
}

\author{
Marco Invernizzi ${ }^{1}$, Letterio Runza ${ }^{2}$, Alessandro De Sire ${ }^{1}$, Lorenzo Lippi ${ }^{1}$, Concetta Blundo ${ }^{3}$, Donatella Gambini ${ }^{4}$, Renzo Boldorini ${ }^{5,6}$, \\ Stefano Ferrero ${ }^{2,7}$, Nicola Fusco ${ }^{2,7}$ \\ ${ }^{1}$ Physical and Rehabilitative Medicine, Department of Health Sciences, University of Eastern Piedmont "A. Avogadro" \\ ${ }^{2}$ Division of Pathology, Fondazione IRCCS Ca' Granda, Ospedale Maggiore Policlinico \\ ${ }^{3}$ Division of Breast Surgery, Fondazione IRCCS Ca' Granda, Ospedale Maggiore Policlinico \\ ${ }^{4}$ Division of Medical Oncology, Fondazione IRCCS Ca' Granda, Ospedale Maggiore Policlinico \\ ${ }^{5}$ Division of Pathology, Maggiore della Carità Hospital \\ ${ }^{6}$ Department of Health Sciences, University of Eastern Piedmont "A. Avogadro" \\ ${ }^{7}$ Department of Biomedical, Surgical, and Dental Sciences, University of Milan
}

Correspondence to: Nicola Fusco at nicola.fusco@unimi.it

URL: https://www.jove.com/video/60093

DOI: doi:10.3791/60093

Keywords: Medicine, Issue 156, breast cancer related lymphedema, breast cancer, 3D laser scanner, Iymphovascular invasion, extranodal extension, axillary lymph nodes dissection

Date Published: 2/6/2020

Citation: Invernizzi, M., Runza, L., De Sire, A., Lippi, L., Blundo, C., Gambini, D., Boldorini, R., Ferrero, S., Fusco, N. Integrating Augmented Reality Tools in Breast Cancer Related Lymphedema Prognostication and Diagnosis. J. Vis. Exp. (156), e60093, doi:10.3791/60093 (2020).

\section{Abstract}

Breast cancer related lymphedema $(B C R L)$ is a detrimental condition characterized by fluid accumulation in the upper limb in breast cancer patients subjected to axillary surgery and/or radiations. Its etiology is multifactorial and include also tumor-specific pathological features, such as lymphovascular invasion (LVI) and extranodal extension (ENE). To date, no widely employed guidelines for the early diagnosis of BCRL are available. Here, we illustrate a protocol for a digitally assisted BCRL assessment using a 3D laser scanner (3DLS) and a tablet computer. It has been specifically optimized in a discovery cohort of high-risk breast cancer patients. This study provides a proof-of-principle that augmented reality tools, such as 3DLS, can be incorporated into the clinical workup of BCRL to allow for a precise, reproducible, reliable, and cheap diagnosis.

\section{Video Link}

The video component of this article can be found at https://www.jove.com/video/60093/

\section{Introduction}

Breast cancer related lymphedema $(B C R L)$ is fluid retention in the upper extremity occurring after axillary surgery and/or radiation therapy in approximately $20-80 \%$ of breast cancer patients with lymph node metastases $(N>1)^{1,2,3}$. This condition results in swelling of the limb with subsequent reduced functionality, increased risk of comorbidities, frustration, anxiety, and depression ${ }^{4,5}$. BCRL is currently considered a longstanding threat for breast cancer survivors given that it can arise up to 9 years after surgery ${ }^{6}$.

BCRL pathogenesis is a subject of lively debate among breast cancer specialists. Hence, several studies have shown that it could be related not only to axillary interventions but also to systemic treatments, such as taxanes-based chemotherapy and anti-HER2 therapy ${ }^{7,8}$. Furthermore, there are recent lines of evidence to suggest that tumor-specific pathological characteristics are involved in its pathogenesis ${ }^{1,6,7}$. For this reason, the invasion of lymphovascular spaces at the periphery of the tumor by neoplastic cells (lymphovascular invasion, LVI) and of the extension of the metastasis beyond the lymph node capsule (extra-nodal extension, ENE) have been proposed as a complementary analysis for BCRL risk stratification $^{6,7}$

Despite the novel insights of BCRL biology, the arm volume measurement remains a cornerstone phase in the diagnosis and follow-up of these patients ${ }^{9}$. For this task, however, there are not widely-adopted guidelines. The measurement of multiple circumferences across the arm has been traditionally used to estimate its total volume using the truncated cone formula ${ }^{10}$. Despite its reliability, however, this circumferential method (CM) lack sensitivity and reproducibility, particularly in the case of arm shape irregularities ${ }^{11}$. Lately, augmented reality methods, such as threedimensional laser scanner (3DLS), have emerged as promising tools for the measurement of the upper limb volume ${ }^{12}$. This technology is cheap, user-friendly, reproducible, and extremely precise also in the presence of gibbousness and swelling ${ }^{2,13}$.

The aim of this study was to assess the reliability of 3DLS in comparison to the circumferential method for BCRL diagnosis and quantification. Here, a protocol is detailed for real-time measurements of the upper limb volume by means of 3DLS in breast cancer patients at increased risk for BCRL. 


\section{Protocol}

This study was approved by the Institutional Review Boards from the Authors' Institutions. This protocol applies to node-positive (i.e., N>1 according to the TNM staging system) ${ }^{14}$ breast cancers showing LVI and/or ENE.

\section{Real time arm measurements}

1. Install the free downloadable application (e.g., Captevia) on a tablet computer.

2. Take a marker of the highest-contrast color compared to the skin of the patient (e.g., white, black).

1. Mark the skin of the upper limb to be analyzed using point-landmarks at $5 \mathrm{~cm}$ starting from wrist to the elbow, and from elbow to proximal portion of the arm. Draw the circumferences on the skin of the upper limb to be analyzed at the level of each landmark.

3. Position the patient in an upright position in a room with sufficient space to move around her/him. Raise the patient's upper limb anteriorly by $90^{\circ}$. Ask the patient to remain still in this position for all the scan duration.

NOTE: In case of difficulty of the patient to maintain this position, a support at hand level (e.g., a stick) can be used.

4. Hook and link the 3DLS device to the tablet.

5. Open the application from the tablet and enter the credentials of the subject to be examined within the patient database.

1. Click Connection and then + to enter a new patient. Enter the patient's Name and Surname together with other preset anamnestic variables (such as date of birth, height, weight, etc.) and others as desired in the dedicated space.

6. Click the 3D icon. Select the area to be examined by modulating the distance and size of the cube that appears on the screen using 2 fingers on the touch screen

7. Click Scan and start the three-dimensional scan framing the upper limb of the patient on all the planes of the space. Rotate around the limb from all the views many times in order to optimize the quality of the acquired image.

NOTE: A good acquisition takes on average time of 30 seconds. Do not stray more than one meter from the limb to be examined. Do not move the tablet too quickly.

8. Once the acquisition is completed, press Done. If needed, revise the form to assess any acquisition defects or missing part.

9. Select the acquisition and press the icon at the bottom right of the screen to send the file. Enter an email to send the file to and click Send.

10. Start the PC and download the data file sent by e-mail. Then insert the USB key with the software license, double click the software icon, confirm selected license type, and open the data file.

11. View the scan and press Create New Patient. Name the patient's file, select the destination to save the file, and press Save.

12. Select File in the upper left corner. Select the patient's file previously named.

13. Select the scan and press Extract to extract the scan. Select the extracted scan and press Cleaning.

14. Select the image. Clean the shape by orienting it in the space ( $x, y$ and $z$ axis). Cut off any segments included in the scan on the various axes $(x, y$ and $z)$. It will also be possible to view any skin marks included in the scan to more precisely cut the scan. Validate the cut figure.

15. Name the shape obtained from the scan. Press Create a new shape.

16. Orient the shape axis on the 3 planes of the space and press End.

17. Select the processed form and press Open: the software will provide the total volume of the processed form.

18. Calculate the volume of different sections, press Volume. Move and select the upper and lower margin of the section from which to extract the volume.

\section{Representative Results}

This was a pilot single-blind, randomized controlled study involving adult individuals. Inclusion criteria were the following: 1) young adults aged $>18$ and <45 years; 2) normal weight (body mass index, BMI, >18 and <25 kg/m ${ }^{2}$ ); 3) absence of any kind of skin lesion at upper limb level; 4 ) absence of trauma and/or any kind of condition able to modify arm structure and volume. The exclusion criteria: 1) cardiovascular comorbidities; 2) vascular diseases involving the upper limb; 3) pathological conditions at thyroid and renal level; 4) presence of lymphedema; 5) previous or active oncological conditions. Moreover, an additional group of women affected by BCRL (stage II-III) referred to the Oncological Rehabilitation unit of the Physical and Rehabilitative Medicine Department of the University Hospital in Novara has been enrolled for a preliminary analysis. Participants were properly informed about the aims of the research, testing procedures, personal data treatment, and the possibility of withdrawal at any time. Written informed consent was obtained from each subject before taking part in the experiment and all the procedures were conducted according to the principles of the Declaration of Helsinki. The study flowchart is represented in Figure 1.

Thirty healthy adults (14 males and 16 females, mean age $27.6 \pm 9.8$ years old), with a mean body mass index (BMI) of $22.7 \pm 2.9 \mathrm{~kg} / \mathrm{m}^{2}(\mathrm{Group}$ A) and 30 breast cancer female patients (mean age $57.9 \pm 13.8$ years old), with a mean BMI of $26.6 \pm 4.6 \mathrm{~kg} / \mathrm{m}^{2}$, who later developed BCRL (Group B) were enrolled from June 2017 to January 2018. The demographic characteristics of the study population are displayed in Table 1. After enrollment, all patients were randomly evaluated by one of the two lynphotherapists with more than 20 years of experience in lymphedema disorder treatments who performed all the experiments. Arm volume measurements were performed using both CM and LS3D methods (Figure 2). Each examiner performed both CM and LS3D upper limb measurement twice for a total of four measurements for each subject (the mean value of the two CM and LS3D evaluations was used). 
In Group A and B, both CM and 3DLS showed high levels of inter- and intra-operator reproducibility, with a mean $r^{2}$ of 0.99 for both techniques (Table 2, Table 3). Accordingly, the mean value of 3DLS volume calculations showed a strong correlation with those from CM in Group A ( ${ }^{2}$ $=0.99 ; p<0.0001)$, as shown in Figure 3a, confirmed by the Bland-Altman plot showing the high level of agreement and the consistency of the two different measurements (Figure $3 b$ ). In Group B, the method showed a strong inter- and intra-operator correlation with $\mathrm{CM}\left(r^{2}=0.99\right.$; $p<0.0001)$. Furthermore, although it was observed a significant $(p<0.001)$ correlation between the inter-operator assessment of total limb volumes differences before and after complex decongestive treatment for both techniques in BCRL women, it is interesting to report that 3DLS correlation was higher than CM $\left(r^{2}=0.85 v s r^{2}=0.62\right)$ (see Figure 3c for further details). Lastly, 3DLS was significantly quicker than CM, with a total time of $202 \pm 27 \mathrm{~s}$ (including acquisition and digital processing) versus $293 \pm 17 \mathrm{~s}(\mathrm{p}<0.0001)$. During the whole study period no CM or 3DLS techniques related drop-outs were recorded in both groups and the Global perceived effect (GPE) ${ }^{15}$ of the 3DLS technique rated on an ordinal 4-point Likert scale showed very good results: 4.5 for the operators and 4.6 for the patients.

\begin{tabular}{|c|c|c|}
\hline & Healthy adults $(n=30)$ & BCRL women $(n=30)$ \\
\hline Age (years) & $27.6 \pm 9.8$ & $57.9 \pm 13.8$ \\
\hline BMI $\left(\mathrm{kg} / \mathrm{m}^{2}\right)$ & $22.7 \pm 2.9$ & $26.6 \pm 4.6$ \\
\hline Sex (female/male) & $16 / 14$ & $30 / 0$ \\
\hline \multicolumn{3}{|l|}{ Level of physical activity $(\mathrm{n}, \%)^{*}$} \\
\hline none & $6(20.0)$ & 7 (23.3) \\
\hline low $w^{a}$ & $10(3.3)$ & $16(53.3)$ \\
\hline medium $^{b}$ & $9(30.0)$ & $5(16.7)$ \\
\hline$h_{i g h}{ }^{c}$ & $5(16.7)$ & $2(6.7)$ \\
\hline Surgery (quadrantectomy/mastectomy) & $0 / 0$ & $17 / 13$ \\
\hline \multicolumn{3}{|c|}{$\begin{array}{l}\text { Continuous variables are expressed as means } \pm \text { standard deviations, categorical variables as counts (percentages), and ratios as } x / y \text {. } \\
\text { Abbreviations: BCRL: breast cancer related lymphedema; BMI = Body Mass Index. }\end{array}$} \\
\hline \multicolumn{3}{|c|}{$\begin{array}{l}\text { *: According to World Health Organization. Global Recommendations on Physical Activity for Health. Geneva, World Health Organization, } 2010 \text {; } \\
\text { a: }<150 \text { minutes of moderate-intensity (or }<75 \text { minutes of vigorous-intensity) aerobic physical activity throughout the week or an equivalent } \\
\text { combination of moderate- and vigorous-intensity activity; b: } 150-300 \text { minutes of moderate-intensity (or } 75-150 \text { minutes of vigorous-intensity) } \\
\text { aerobic physical activity throughout the week or an equivalent combination of moderate- and vigorous-intensity activity; c: }>300 \text { minutes of } \\
\text { moderate-intensity (or }>150 \text { minutes of vigorous-intensity) aerobic physical activity throughout the week or an equivalent combination of moderate- } \\
\text { and vigorous-intensity activity. }\end{array}$} \\
\hline
\end{tabular}

Table 1: Anamnestic and demographical characteristics of study population. 


\begin{tabular}{|c|c|c|c|c|c|c|c|c|c|}
\hline & \multicolumn{9}{|c|}{ Healthy adults $(n=30)$} \\
\hline & \multicolumn{4}{|c|}{ Intra-operator analysis } & & & \multicolumn{2}{|c|}{ Inter-operator analysis } & \\
\hline & \multicolumn{3}{|c|}{ Operator A } & \multicolumn{3}{|l|}{ Operator B } & \multirow{2}{*}{$\begin{array}{l}\text { Operator } \\
A \\
\text { Mean }\end{array}$} & \multirow{2}{*}{\begin{tabular}{|l|} 
Operator B \\
Mean \\
\end{tabular}} & \\
\hline & $\begin{array}{l}1^{\text {st }} \\
\text { measure }\end{array}$ & $2^{\text {nd }}$ measure & $r^{2}$ & $1^{\text {st }}$ measure & $\begin{array}{l}2^{\text {nd }} \\
\text { measure }\end{array}$ & $r^{2}$ & & & $r^{2}$ \\
\hline $\mathrm{V} 1\left(\mathrm{dm}^{3}\right)$ & $\begin{array}{l}0.14 \pm \\
0.05\end{array}$ & $0.14 \pm 0.05$ & 0.99 & $0.14 \pm 0.05$ & $\begin{array}{l}0.14 \pm \\
0.05\end{array}$ & 0.99 & $\begin{array}{l}0.14 \pm \\
0.05\end{array}$ & $0.14 \pm 0.05$ & 0.99 \\
\hline $\mathrm{V} 2\left(\mathrm{dm}^{3}\right)$ & $\begin{array}{l}0.15 \pm \\
0.04\end{array}$ & $0.14 \pm 0.04$ & 0.98 & $0.15 \pm 0.04$ & $\begin{array}{l}0.15 \pm \\
0.04\end{array}$ & 0.99 & $\begin{array}{l}0.15 \pm \\
0.04\end{array}$ & $0.15 \pm 0.04$ & 0.99 \\
\hline V3 $\left(\mathrm{dm}^{3}\right)$ & $\begin{array}{l}0.21 \pm \\
0.05\end{array}$ & $0.21 \pm 0.05$ & 0.99 & $0.21 \pm 0.05$ & $\begin{array}{l}0.21 \pm \\
0.05\end{array}$ & 0.99 & $\begin{array}{l}0.21 \pm \\
0.05\end{array}$ & $0.21 \pm 0.05$ & 0.99 \\
\hline $\mathrm{V} 4\left(\mathrm{dm}^{3}\right)$ & $\begin{array}{l}0.26 \pm \\
0.06\end{array}$ & $0.26 \pm 0.06$ & 0.99 & $0.26 \pm 0.06$ & $\begin{array}{l}0.26 \pm \\
0.06\end{array}$ & 0.99 & $\begin{array}{l}0.26 \pm \\
0.06\end{array}$ & $0.26 \pm 0.06$ & 0.99 \\
\hline V5 $\left(\mathrm{dm}^{3}\right)$ & $\begin{array}{l}0.27 \pm \\
0.06\end{array}$ & $0.27 \pm 0.06$ & 0.99 & $0.27 \pm 0.05$ & $\begin{array}{l}0.27 \pm \\
0.05\end{array}$ & 0.99 & $\begin{array}{l}0.27 \pm \\
0.06\end{array}$ & $0.27 \pm 0.05$ & 0.99 \\
\hline $\mathrm{V} 6\left(\mathrm{dm}^{3}\right)$ & $\begin{array}{l}0.27 \pm \\
0.06\end{array}$ & $0.27 \pm 0.06$ & 0.99 & $0.27 \pm 0.06$ & $\begin{array}{l}0.27 \pm \\
0.06\end{array}$ & 0.99 & $\begin{array}{l}0.27 \pm \\
0.06\end{array}$ & $0.27 \pm 0.06$ & 0.99 \\
\hline$V 7\left(d^{3}\right)$ & $\begin{array}{l}0.30 \pm \\
0.09\end{array}$ & $0.30 \pm 0.09$ & 0.99 & $0.30 \pm 0.09$ & $\begin{array}{l}0.30 \pm \\
0.09\end{array}$ & 0.99 & $\begin{array}{l}0.30 \pm \\
0.09\end{array}$ & $0.30 \pm 0.09$ & 0.99 \\
\hline $\mathrm{V} 8\left(\mathrm{dm}^{3}\right)$ & $\begin{array}{l}0.35 \pm \\
0.11\end{array}$ & $0.35 \pm 0.11$ & 0.99 & $0.35 \pm 0.11$ & $\begin{array}{l}0.35 \pm \\
0.11\end{array}$ & 0.99 & $\begin{array}{l}0.35 \pm \\
0.11\end{array}$ & $0.35 \pm 0.11$ & 0.99 \\
\hline $\begin{array}{l}V \text { forearm } \\
\left(\mathrm{dm}^{3}\right)\end{array}$ & $\begin{array}{l}1.04 \pm \\
0.24\end{array}$ & $1.04 \pm 0.24$ & 0.99 & $1.04 \pm 0.24$ & $\begin{array}{l}1.04 \pm \\
0.24\end{array}$ & 0.99 & $\begin{array}{l}1.04 \pm \\
0.24\end{array}$ & $1.04 \pm 0.24$ & 0.99 \\
\hline $\begin{array}{l}\text { V arm } \\
\left(\mathrm{dm}^{3}\right)\end{array}$ & $\begin{array}{l}0.92 \pm \\
0.26\end{array}$ & $0.92 \pm 0.26$ & 0.99 & $0.92 \pm 0.26$ & $\begin{array}{l}0.92 \pm \\
0.26\end{array}$ & 0.99 & $\begin{array}{l}0.92 \pm \\
0.26\end{array}$ & $0.92 \pm 0.26$ & 0.99 \\
\hline \multirow[t]{5}{*}{$\begin{array}{l}V \text { tot } \\
\left(\mathrm{dm}^{3}\right)\end{array}$} & $\begin{array}{l}1.96 \pm \\
0.47\end{array}$ & $1.96 \pm 0.47$ & 0.99 & $1.96 \pm 0.47$ & $\begin{array}{l}1.96 \pm \\
0.47\end{array}$ & 0.99 & $\begin{array}{l}1.96 \pm \\
0.47\end{array}$ & $1.96 \pm 0.47$ & 0.99 \\
\hline & \multicolumn{9}{|c|}{ BCRL women $(n=30)$} \\
\hline & \multicolumn{4}{|c|}{ Intra-operator analysis } & & & \multicolumn{2}{|c|}{ Inter-operator analysis } & \\
\hline & \multicolumn{3}{|c|}{ Operator A } & \multicolumn{3}{|l|}{ Operator B } & $\begin{array}{l}\text { Operator } \\
\text { A }\end{array}$ & Operator B & \\
\hline & $\begin{array}{l}1^{\text {st }} \\
\text { measure }\end{array}$ & $2^{\text {nd }}$ measure & $r^{2}$ & $1^{\text {st }}$ measure & $\begin{array}{l}2^{\text {nd }} \\
\text { measure }\end{array}$ & $r^{2}$ & Mean & Mean & $r^{2}$ \\
\hline $\mathrm{V} 1\left(\mathrm{dm}^{3}\right)$ & $\begin{array}{l}0.14 \pm \\
0.05\end{array}$ & $0.14 \pm 0.05$ & 0.99 & $0.14 \pm 0.05$ & $\begin{array}{l}0.14 \pm \\
0.05\end{array}$ & 0.99 & $\begin{array}{l}0.14 \pm \\
0.05\end{array}$ & $0.14 \pm 0.05$ & 0.98 \\
\hline $\mathrm{V} 2\left(\mathrm{dm}^{3}\right)$ & $\begin{array}{l}0.20 \pm \\
0.08\end{array}$ & $0.20 \pm 0.08$ & 0.99 & $0.20 \pm 0.08$ & $\begin{array}{l}0.20 \pm \\
0.08\end{array}$ & 0.99 & $\begin{array}{l}0.20 \pm \\
0.08\end{array}$ & $0.20 \pm 0.08$ & 0.99 \\
\hline V3 $\left(\mathrm{dm}^{3}\right)$ & $\begin{array}{l}0.27 \pm \\
0.10\end{array}$ & $0.27 \pm 0.10$ & 0.99 & $0.27 \pm 0.10$ & $\begin{array}{l}0.27 \pm \\
0.10\end{array}$ & 0.99 & $\begin{array}{l}0.27 \pm \\
0.10\end{array}$ & $0.27 \pm 0.10$ & 0.99 \\
\hline $\mathrm{V} 4\left(\mathrm{dm}^{3}\right)$ & $\begin{array}{l}0.34 \pm \\
0.10\end{array}$ & $0.34 \pm 0.10$ & 0.99 & $0.34 \pm 0.10$ & $\begin{array}{l}0.34 \pm \\
0.10\end{array}$ & 0.99 & $\begin{array}{l}0.34 \pm \\
0.10\end{array}$ & $0.34 \pm 0.10$ & 0.99 \\
\hline $\mathrm{V} 5\left(\mathrm{dm}^{3}\right)$ & $\begin{array}{l}0.36 \pm \\
0.10\end{array}$ & $0.36 \pm 0.10$ & 0.99 & $0.36 \pm 0.10$ & $\begin{array}{l}0.36 \pm \\
0.10\end{array}$ & 0.99 & $\begin{array}{l}0.36 \pm \\
0.10\end{array}$ & $0.36 \pm 0.10$ & 0.99 \\
\hline V6 $\left(\mathrm{dm}^{3}\right)$ & $\begin{array}{l}0.38 \pm \\
0.11\end{array}$ & $0.38 \pm 0.11$ & 0.99 & $0.38 \pm 0.11$ & $\begin{array}{l}0.38 \pm \\
0.11\end{array}$ & 0.99 & $\begin{array}{l}0.38 \pm \\
0.11\end{array}$ & $0.38 \pm 0.11$ & 0.98 \\
\hline$V 7\left(\mathrm{dm}^{3}\right)$ & $\begin{array}{l}0.44 \pm \\
0.12\end{array}$ & $0.44 \pm 0.12$ & 0.99 & $0.44 \pm 0.12$ & $\begin{array}{l}0.44 \pm \\
0.12\end{array}$ & 0.99 & $\begin{array}{l}0.44 \pm \\
0.12\end{array}$ & $0.44 \pm 0.12$ & 0.99 \\
\hline $\mathrm{V} 8\left(\mathrm{dm}^{3}\right)$ & $\begin{array}{l}0.48 \pm \\
0.15\end{array}$ & $0.48 \pm 0.15$ & 0.99 & $0.48 \pm 0.16$ & $\begin{array}{l}0.48 \pm \\
0.16\end{array}$ & 0.99 & $\begin{array}{l}0.48 \pm \\
0.16\end{array}$ & $0.48 \pm 0.16$ & 0.99 \\
\hline $\begin{array}{l}V \text { forearm } \\
\left(\mathrm{dm}^{3}\right)\end{array}$ & $\begin{array}{l}1.30 \pm \\
0.41\end{array}$ & $1.30 \pm 0.41$ & 0.99 & $1.31 \pm 0.41$ & $\begin{array}{l}1.31 \pm \\
0.41\end{array}$ & 0.99 & $\begin{array}{l}1.31 \pm \\
0.41\end{array}$ & $1.31 \pm 0.41$ & 0.99 \\
\hline
\end{tabular}




\begin{tabular}{|l|l|l|l|l|l|l|l|l|l|}
\hline $\begin{array}{l}\text { V arm } \\
\left(\mathrm{dm}^{3}\right)\end{array}$ & $\begin{array}{l}1.29 \pm \\
0.38\end{array}$ & $1.29 \pm 0.38$ & 0.99 & $1.30 \pm 0.38$ & $\begin{array}{l}1.30 \pm \\
0.38\end{array}$ & 0.99 & $\begin{array}{l}1.30 \pm \\
0.38\end{array}$ & $1.30 \pm 0.38$ \\
\hline $\begin{array}{l}\text { V tot } \\
\left(\mathrm{dm}^{3}\right)\end{array}$ & $\begin{array}{l}2.60 \pm \\
0.77\end{array}$ & $2.60 \pm 0.77$ & 0.99 & $2.61 \pm 0.76$ & $\begin{array}{l}2.61 \pm \\
0.76\end{array}$ & 0.99 & $\begin{array}{l}2.61 \pm \\
0.76\end{array}$ & $2.61 \pm 0.76$ \\
\hline
\end{tabular}

Data are expressed as means \pm standard deviations. Abbreviations: CM: circumferential method; BCRL: breast cancer related lymphedema; $r^{2}$ :

Pearson's $\mathrm{r}^{2}$; V: volume.

Table 2: Intra- and inter-operator analysis of circumferential method in healthy adults and BCRL women. 


\begin{tabular}{|c|c|c|c|c|c|c|c|c|c|}
\hline & \multicolumn{9}{|c|}{ Healthy adults $(n=30)$} \\
\hline & \multicolumn{4}{|c|}{ Intra-operator analysis } & & & \multicolumn{2}{|c|}{ Inter-operator analysis } & \\
\hline & \multicolumn{3}{|c|}{ Operator A } & \multicolumn{3}{|l|}{ Operator B } & \multirow{2}{*}{\begin{tabular}{|l|}
$\begin{array}{l}\text { Operator } \\
A\end{array}$ \\
Mean \\
\end{tabular}} & \multirow{2}{*}{\begin{tabular}{|l|} 
Operator B \\
Mean \\
\end{tabular}} & \\
\hline & $\begin{array}{l}1^{\text {st }} \\
\text { measure }\end{array}$ & $2^{\text {nd }}$ measure & $r^{2}$ & $1^{\text {st }}$ measure & $\begin{array}{l}2^{\text {nd }} \\
\text { measure }\end{array}$ & $r^{2}$ & & & $r^{2}$ \\
\hline $\mathrm{V} 1\left(\mathrm{dm}^{3}\right)$ & $\begin{array}{l}0.13 \pm \\
0.05\end{array}$ & $0.13 \pm 0.05$ & 0.99 & $0.14 \pm 0.06$ & $\begin{array}{l}0.14 \pm \\
0.06\end{array}$ & 0.99 & $\begin{array}{l}0.13 \pm \\
0.05\end{array}$ & $0.14 \pm 0.06$ & 0.99 \\
\hline $\mathrm{V} 2\left(\mathrm{dm}^{3}\right)$ & $\begin{array}{l}0.16 \pm \\
0.04\end{array}$ & $0.16 \pm 0.04$ & 0.99 & $0.16 \pm 0.04$ & $\begin{array}{l}0.16 \pm \\
0.05\end{array}$ & 0.99 & $\begin{array}{l}0.16 \pm \\
0.04\end{array}$ & $0.16 \pm 0.04$ & 0.99 \\
\hline$V 3\left(\mathrm{dm}^{3}\right)$ & $\begin{array}{l}0.21 \pm \\
0.05\end{array}$ & $0.20 \pm 0.05$ & 0.99 & $0.21 \pm 0.06$ & $\begin{array}{l}0.22 \pm \\
0.06\end{array}$ & 0.99 & $\begin{array}{l}0.21 \pm \\
0.05\end{array}$ & $0.21 \pm 0.06$ & 0.99 \\
\hline $\mathrm{V} 4\left(\mathrm{dm}^{3}\right)$ & $\begin{array}{l}0.25 \pm \\
0.06\end{array}$ & $0.25 \pm 0.06$ & 0.99 & $0.25 \pm 0.06$ & $\begin{array}{l}0.25 \pm \\
0.06\end{array}$ & 0.99 & $\begin{array}{l}0.25 \pm \\
0.06\end{array}$ & $0.25 \pm 0.06$ & 0.99 \\
\hline $\mathrm{V} 5\left(\mathrm{dm}^{3}\right)$ & $\begin{array}{l}0.26 \pm \\
0.05\end{array}$ & $0.26 \pm 0.05$ & 0.99 & $0.26 \pm 0.06$ & $\begin{array}{l}0.26 \pm \\
0.06\end{array}$ & 0.99 & $\begin{array}{l}0.26 \pm \\
0.05\end{array}$ & $0.26 \pm 0.06$ & 0.99 \\
\hline V6 $\left(\mathrm{dm}^{3}\right)$ & $\begin{array}{l}0.27 \pm \\
0.07\end{array}$ & $0.27 \pm 0.07$ & 0.99 & $0.27 \pm 0.07$ & $\begin{array}{l}0.27 \pm \\
0.07\end{array}$ & 0.99 & $\begin{array}{l}0.27 \pm \\
0.07\end{array}$ & $0.27 \pm 0.07$ & 0.99 \\
\hline$V 7\left(\mathrm{dm}^{3}\right)$ & $\begin{array}{l}0.30 \pm \\
0.09\end{array}$ & $0.30 \pm 0.09$ & 0.99 & $0.30 \pm 0.09$ & $\begin{array}{l}0.31 \pm \\
0.09\end{array}$ & 0.99 & $\begin{array}{l}0.30 \pm \\
0.09\end{array}$ & $0.30 \pm 0.09$ & 0.99 \\
\hline $\mathrm{V} 8\left(\mathrm{dm}^{3}\right)$ & $\begin{array}{l}0.33 \pm \\
0.10\end{array}$ & $0.32 \pm 0.10$ & 0.99 & $0.33 \pm 0.10$ & $\begin{array}{l}0.33 \pm \\
0.10\end{array}$ & 0.99 & $\begin{array}{l}0.33 \pm \\
0.10\end{array}$ & $0.33 \pm 0.10$ & 0.99 \\
\hline $\begin{array}{l}V \text { forearm } \\
\left(\mathrm{dm}^{3}\right)\end{array}$ & $\begin{array}{l}1.02 \pm \\
0.25\end{array}$ & $1.02 \pm 0.25$ & 0.99 & $1.02 \pm 0.25$ & $\begin{array}{l}1.02 \pm \\
0.25\end{array}$ & 0.99 & $\begin{array}{l}1.02 \pm \\
0.25\end{array}$ & $1.02 \pm 0.25$ & 0.99 \\
\hline $\begin{array}{l}\text { V arm } \\
\left(\mathrm{dm}^{3}\right)\end{array}$ & $\begin{array}{l}0.89 \pm \\
0.26\end{array}$ & $0.89 \pm 0.26$ & 0.99 & $0.90 \pm 0.26$ & $\begin{array}{l}0.90 \pm \\
0.26\end{array}$ & 0.99 & $\begin{array}{l}0.89 \pm \\
0.26\end{array}$ & $0.90 \pm 0.26$ & 0.99 \\
\hline \multirow[t]{5}{*}{$\begin{array}{l}V \text { tot } \\
\left(\mathrm{dm}^{3}\right)\end{array}$} & $\begin{array}{l}1.91 \pm \\
0.48\end{array}$ & $1.91 \pm 0.48$ & 0.99 & $1.91 \pm 0.49$ & $\begin{array}{l}1.91 \pm \\
0.49\end{array}$ & 0.99 & $\begin{array}{l}1.91 \pm \\
0.48\end{array}$ & $1.91 \pm 0.49$ & 0.99 \\
\hline & \multicolumn{9}{|c|}{$B C R L$ women $(n=30)$} \\
\hline & \multicolumn{4}{|c|}{ Intra-operator analysis } & & & \multicolumn{2}{|c|}{ Inter-operator analysis } & \\
\hline & \multicolumn{3}{|c|}{ Operator A } & \multicolumn{3}{|l|}{ Operator B } & $\begin{array}{l}\text { Operator } \\
\text { A }\end{array}$ & Operator B & \\
\hline & $\begin{array}{l}1^{\text {st }} \\
\text { measure }\end{array}$ & $2^{\text {nd }}$ measure & $r^{2}$ & $1^{\text {st }}$ measure & $\begin{array}{l}2^{\text {nd }} \\
\text { measure }\end{array}$ & $r^{2}$ & Mean & Mean & $r^{2}$ \\
\hline $\mathrm{V} 1\left(\mathrm{dm}^{3}\right)$ & $\begin{array}{l}0.08 \pm \\
0.06\end{array}$ & $0.08 \pm 0.06$ & 0.99 & $0.08 \pm 0.06$ & $\begin{array}{l}0.08 \pm \\
0.06\end{array}$ & 0.99 & $\begin{array}{l}0.08 \pm \\
0.06\end{array}$ & $0.08 \pm 0.06$ & 0.99 \\
\hline $\mathrm{V} 2\left(\mathrm{dm}^{3}\right)$ & $\begin{array}{l}0.20 \pm \\
0.09\end{array}$ & $0.20 \pm 0.09$ & 0.99 & $0.20 \pm 0.09$ & $\begin{array}{l}0.20 \pm \\
0.09\end{array}$ & 0.99 & $\begin{array}{l}0.20 \pm \\
0.09\end{array}$ & $0.20 \pm 0.09$ & 0.99 \\
\hline V3 $\left(\mathrm{dm}^{3}\right)$ & $\begin{array}{l}0.28 \pm \\
0.10\end{array}$ & $0.28 \pm 0.10$ & 0.99 & $0.28 \pm 0.10$ & $\begin{array}{l}0.28 \pm \\
0.10\end{array}$ & 0.99 & $\begin{array}{l}0.28 \pm \\
0.10\end{array}$ & $0.28 \pm 0.10$ & 0.99 \\
\hline $\mathrm{V} 4\left(\mathrm{dm}^{3}\right)$ & $\begin{array}{l}0.34 \pm \\
0.11\end{array}$ & $0.34 \pm 0.11$ & 0.99 & $0.34 \pm 0.11$ & $\begin{array}{l}0.34 \pm \\
0.11\end{array}$ & 0.99 & $\begin{array}{l}0.34 \pm \\
0.11\end{array}$ & $0.34 \pm 0.11$ & 0.99 \\
\hline $\mathrm{V} 5\left(\mathrm{dm}^{3}\right)$ & $\begin{array}{l}0.35 \pm \\
0.10\end{array}$ & $0.35 \pm 0.10$ & 0.99 & $0.35 \pm 0.10$ & $\begin{array}{l}0.35 \pm \\
0.10\end{array}$ & 0.99 & $\begin{array}{l}0.35 \pm \\
0.10\end{array}$ & $0.35 \pm 0.10$ & 0.99 \\
\hline V6 $\left(\mathrm{dm}^{3}\right)$ & $\begin{array}{l}0.37 \pm \\
0.11\end{array}$ & $0.37 \pm 0.11$ & 0.99 & $0.37 \pm 0.11$ & $\begin{array}{l}0.37 \pm \\
0.11\end{array}$ & 0.99 & $0.37 \pm$ & $0.37 \pm 0.11$ & 0.98 \\
\hline $\mathrm{V} 7\left(\mathrm{dm}^{3}\right)$ & $\begin{array}{l}0.43 \pm \\
0.12\end{array}$ & $0.43 \pm 0.12$ & 0.99 & $0.43 \pm 0.12$ & $\begin{array}{l}0.43 \pm \\
0.12\end{array}$ & 0.99 & $\begin{array}{l}0.43 \pm \\
0.12\end{array}$ & $0.43 \pm 0.12$ & 0.99 \\
\hline $\mathrm{V} 8\left(\mathrm{dm}^{3}\right)$ & $\begin{array}{l}0.46 \pm \\
0.15\end{array}$ & $0.46 \pm 0.15$ & 0.99 & $0.46 \pm 0.15$ & $\begin{array}{l}0.46 \pm \\
0.15\end{array}$ & 0.99 & $\begin{array}{l}0.46 \pm \\
0.15\end{array}$ & $0.46 \pm 0.15$ & 0.99 \\
\hline $\begin{array}{l}V \text { forearm } \\
\left(\mathrm{dm}^{3}\right)\end{array}$ & $\begin{array}{l}1.31 \pm \\
0.45\end{array}$ & $1.31 \pm 0.45$ & 0.99 & $1.32 \pm 0.45$ & $\begin{array}{l}1.32 \pm \\
0.45\end{array}$ & 0.99 & $\begin{array}{l}1.32 \pm \\
0.45\end{array}$ & $1.32 \pm 0.45$ & 0.99 \\
\hline
\end{tabular}




\begin{tabular}{|l|l|l|l|l|l|l|l|l|l|}
\hline $\begin{array}{l}\text { V arm } \\
\left(\mathrm{dm}^{3}\right)\end{array}$ & $\begin{array}{l}1.26 \pm \\
0.37\end{array}$ & $1.26 \pm 0.37$ & 0.99 & $1.27 \pm 0.37$ & $\begin{array}{l}1.27 \pm \\
0.37\end{array}$ & 0.99 & $\begin{array}{l}1.27 \pm \\
0.37\end{array}$ & $1.27 \pm 0.37$ \\
\hline $\begin{array}{l}\text { V tot } \\
\left(\mathrm{dm}^{3}\right)\end{array}$ & $\begin{array}{l}2.58 \pm \\
0.79\end{array}$ & $2.58 \pm 0.79$ & 0.99 & $2.59 \pm 0.79$ & $\begin{array}{l}2.59 \pm \\
0.79\end{array}$ & 0.99 & $\begin{array}{l}2.59 \pm \\
0.79\end{array}$ & $2.59 \pm 0.79$ \\
\hline
\end{tabular}

Data are expressed as means \pm standard deviations. Abbreviations: LS3D three-dimensional laser scanning; BCRL: breast cancer related lymphedema; $r^{2}$ : Pearson's $r^{2}$; V: volume.

Table 3: Intra- and inter-operator analysis of laser scanning 3D in healthy adults and BCRL women.

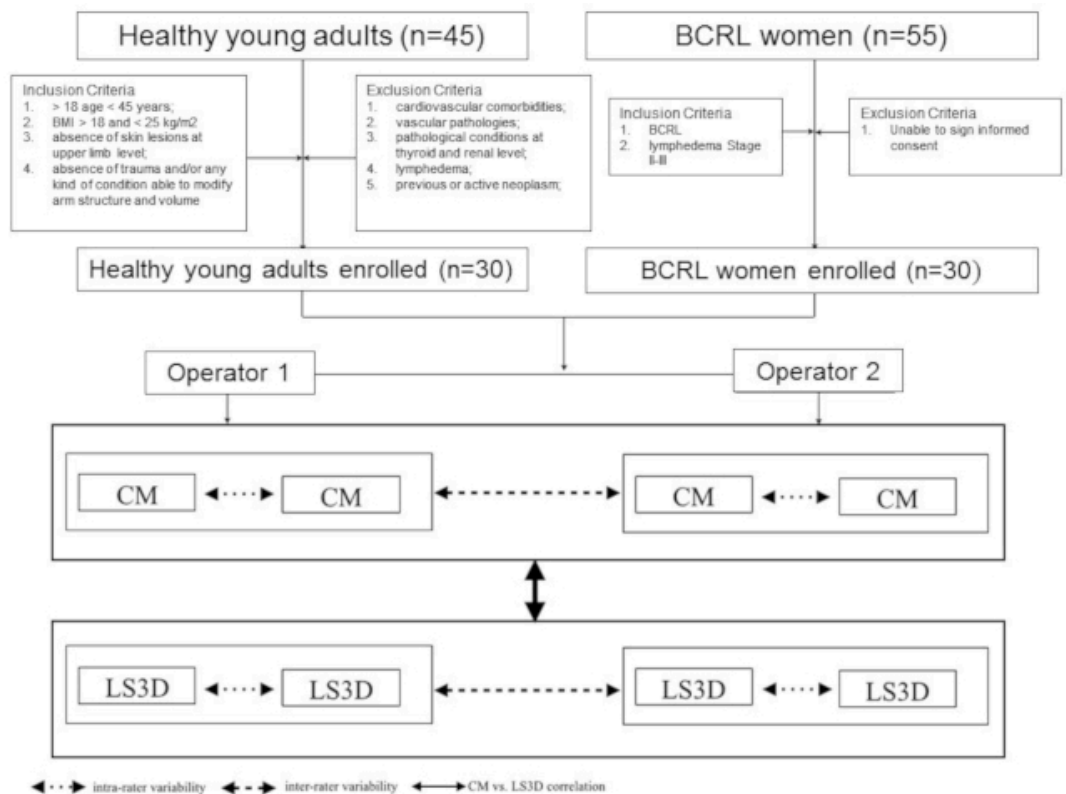

Figure 1: Study flow chart. Please click here to view a larger version of this figure.

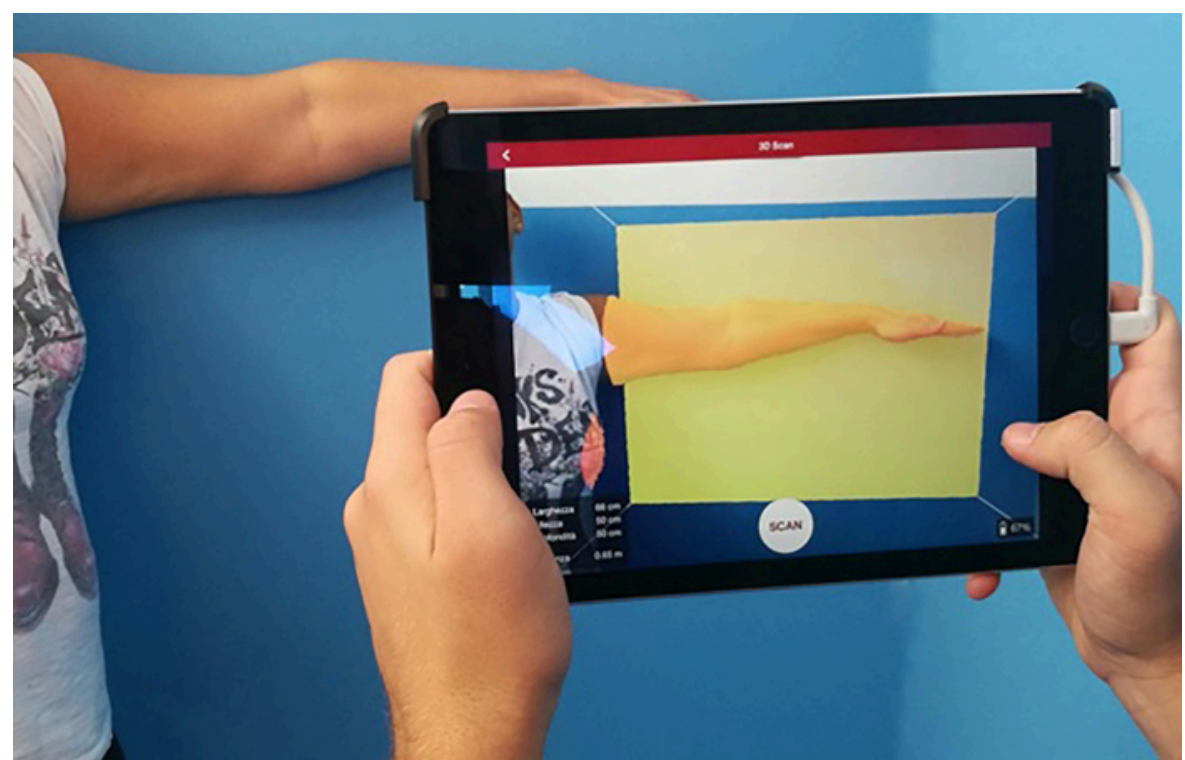

Figure 2: Three-dimensional laser scanning of the upper limb in BCRL. Please click here to view a larger version of this figure. 
a

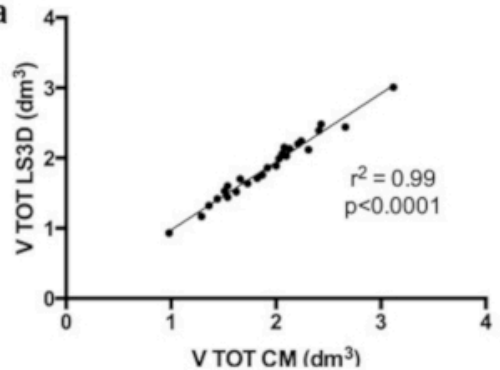

c

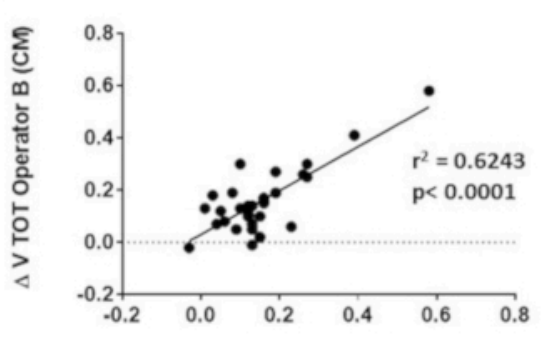

$\Delta \mathrm{V}$ TOT Operator $\mathrm{A}(\mathrm{CM})$ b
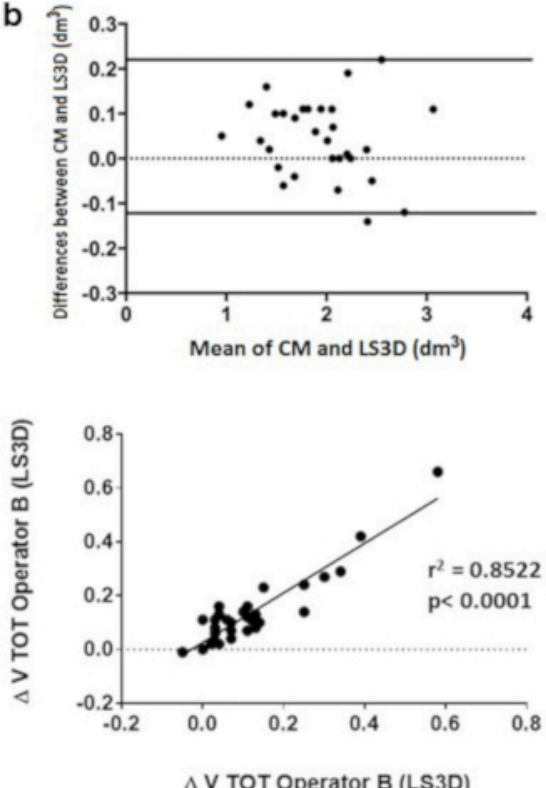

$\triangle \mathrm{V}$ TOT Operator B (LS3D)

Figure 3: Comparative analyses between the laser scanning 3D and circumferential method. (a) Correlation between laser scanning 3D and circumferential method; (b) Bland-Altman plot showing the level of agreement and the consistency of the different measurement; (c) Correlation between the inter-rater operator analysis in terms of total limb volume differences before and after rehabilitative treatment of both circumferential method and laser scanning 3D in breast cancer related lymphedema patients. Please click here to view a larger version of this figure.

\section{Discussion}

The use of an accurate and reliable tool for limb volume measurement is mandatory in breast cancer survivors, given that an early diagnosis of $B C R L$ is related to improved outcomes. In addition, the identification of high-risk individuals should consider not only clinical and surgical data but also tumor-specific pathological features. This study demonstrates the reproducibility and reliability of a new portable 3DLS device in upper limb volume measurement both in healthy subjects and in a BCRL women with high-risk features. This method is safe, painless, cost-effective and can be carried out in most Institutions in large cohorts of patients.

The purpose of a 3D scanner is to create a point cloud of geometric shapes on the surface of the subject, which represent the 3D model. In particular, the 3DLS technology employs a triangulation mechanism projecting a laser dot into an object (in this case, the upper limb), then the sensor measures the distance to the surface of the object. This new standardized augmented reality-based protocol shows that 3DLS is a promising technique for quick volume measurement, not only in sanitary settings. Results obtained in both healthy subjects and BCRL patients are intriguing considering the relative quickness, accuracy and reproducibility of this device. One of the main critical points of this protocol is related to the 3DLS acquisition procedure which can lead to incomplete/incorrect images or errors in upper limb volumes quantification. To overcome this inconvenience, the acquisition in a room with ample free spaces is recommended, as well as to ask the patient to remain still with the upper limb anteriorly raised by $90^{\circ}$ for all the scan duration to avoid inaccuracies in the volumetric detection. Moreover, in order to obtain better volume acquisitions and to optimize the quality of the acquired image it is recommended to rotate the 3DLS around the limb from all the views many times. The acquisition should end only when the entire volumetric image of the upper limb has been acquired and the mean acquisition time is about $30 \mathrm{~s}$. However, despite these potential drawbacks related to the acquisition procedure, these data about failed volume measure attempts suggest a relatively fast learning curve of 3DLS structure sensor, at least comparable to CM. Taken together all these points suggest important implications for the implementation of portable 3DLS techniques in different real-life clinical settings (i.e., outpatient or home care) and for the potential reduction of health sanitary costs in terms of both personnel and procedures costs. Therefore, structure sensor implementation in both diagnostic and therapeutic setting could have significant clinical implications for lymphedema management and rehabilitation confirming the capital role of augmented reality technologies in BCRL clinical workup, where reliable screening, diagnostic, and follow-up tools are needed to achieve the goal of precision medicine. It is important to note, however, that this protocol provide substantial improvements in the workup of BCRL only for selected groups of BCRL patients, particularly in the setting of an initial assessment. Hence, many patients with lymphedema unfortunately arrive at a treatment center with significant levels of swelling and often with comorbid conditions such as obesity. Furthermore, it must also be noted that the $3 \mathrm{D}$ scanning was always performed after the CM. The difference in acquisition time may reflect a learning curve. Another limitation of this study is represented by the randomization of the control group, where no matching for the age of the patients was made. On the other hand, this pilot study should be considered a proof-of-principle.

In conclusion, the paradigm-shift of using augmented reality tools in $\mathrm{N}>1$ breast cancer patients, coupled with a tumor-based risk stratification would represent a step toward precision medicine in BCRL clinical management. 


\section{Disclosures}

None.

\section{Acknowledgments}

None.

\section{References}

1. Michelotti, A. et al. Tackling the diversity of breast cancer related lymphedema: Perspectives on diagnosis, risk assessment, and clinical management. Breast. 44, 15-23 (2018).

2. Noguchi, M., Yokoi, M., Nakano, Y., Ohno, Y., Kosaka, T. Axillary reverse mapping in breast cancer. In: Singh N, ed. Radioisotopes Applications in Bio-Medical Science. Rijeka, Croatia: InTech Publisher (2011).

3. Wilke, L. G. et al. Surgical complications associated with sentinel lymph node biopsy: results from a prospective international cooperative group trial. Annals of Surgical Oncology. 13 (4), 491-500 (2006).

4. Taghian, N. R., Miller, C. L., Jammallo, L. S., O'Toole, J., Skolny, M. N. Lymphedema following breast cancer treatment and impact on quality of life: a review. Critical Reviews in Oncology/Hematology. 92 (3), 227-234 (2014).

5. Dean, L. T. et al. "It still affects our economic situation": long-term economic burden of breast cancer and lymphedema. Supportive Care in Cancer. (2018)

6. Invernizzi, M. et al. Lymphovascular invasion and extranodal tumour extension are risk indicators of breast cancer related lymphoedema: an observational retrospective study with long-term follow-up. BMC Cancer. 18 (1), 935 (2018).

7. Invernizzi, M. et al. Breast Cancer Systemic Treatments and Upper Limb Lymphedema: A Risk-Assessment Platform Encompassing TumorSpecific Pathological Features Reveals the Potential Role of Trastuzumab. Journal of Clinical Medicine. 8 (2) (2019).

8. Kilbreath, S. L. et al. Risk factors for lymphoedema in women with breast cancer: A large prospective cohort. Breast. 28, 29-36 (2016).

9. Sun, F. et al. The need for preoperative baseline arm measurement to accurately quantify breast cancer-related lymphedema. Breast Cancer Research and Treatment. 157 (2), 229-240 (2016).

10. Deltombe, T. et al. Reliability and limits of agreement of circumferential, water displacement, and optoelectronic volumetry in the measurement of upper limb lymphedema. Lymphology. 40 (1), 26-34 (2007).

11. Tewari, N., Gill, P. G., Bochner, M. A., Kollias, J. Comparison of volume displacement versus circumferential arm measurements for lymphoedema: implications for the SNAC trial. ANZ Journal of Surgery. 78 (10), 889-893 (2008).

12. Cau, N. et al. Comparative study between circumferential method and laser scanner 3D method for the evaluation of arm volume in healthy subjects. Journal of Vascular Surgery: Venous and Lymphatic Disorders. 4 (1), 64-72 (2016).

13. Hameeteman, M., Verhulst, A. C., Vreeken, R. D., Maal, T. J., Ulrich, D. J. 3D stereophotogrammetry in upper-extremity lymphedema: An accurate diagnostic method. Journal of Plastic, Reconstructive \& Aesthetic Surgery. 69 (2), 241-247 (2016).

14. Amin, M. B. et al. AJCC Cancer Staging Manual. Eighth Edition, Springer International Publishing, (2017).

15. Kamper, S. J. et al. Global Perceived Effect scales provided reliable assessments of health transition in people with musculoskeletal disorders, but ratings are strongly influenced by current status. Journal of Clinical Epidemiology. 63 (7), 760-766.e761 (2010). 